\section{Método dental modificado para la estimación de la edad en individuos adultos}

\author{
Dental modified method for estimating age in adult individuals
}

\section{Resumen}

El objetivo principal de la investigación fue contrastar el método dental modifcado para la estimación de la edad basados en los métodos de Lamendin, Prince - Ubelaker y Ubelaker - Parra, en individuos adultos de Lima Metropolitana. La muestra consistió en 50 dientes sanos, permanentes y uniradiculares de pacientes adultos de 25 a 75 años atendidos en diferentes Centros de Salud de Lima Metropolitana. Se validó la aplicabilidad de tres métodos dentales (Lamendin, Prince - Ubelaker y Ubelaker-Parra) para estimar la edad en la muestra del estudio. Para ello se aplicó el test de Wilcoxon el cual demostró que las edades estimadas tanto por el método de Prince - Ubelaker como el de Ubelaker - Parra no difirieron significativamente de las edades reales, en comparación con los resultados de Lamendin que si presentaron diferencia significativa. La estimación fue mejor en el caso del método de Prince - Ubelaker $(\mathrm{r}=0.996)$, comparado con el de Ubelaker - Parra $(\mathrm{r}=0.889)$, siendo el primero el más preciso en la estimación de la edad para la muestra del estudio. Aplicando la prueba de regresión múltiple sobre el modelo de fórmula de Prince - Ubelaker se pudo obtener una fórmula, la cual fue muy precisa para la estimación de la edad en la muestra de individuos adultos de Lima Metropolitana. Se concluye que los métodos de Prince - Ubelaker y Ubelaker - Parra son válidos para estimar la edad en individuos adultos de Lima Metropolitana, además que fue factible desarrollar un método dental específico para estimar con mayor precisión la edad en una muestra de individuos adultos de Lima Metropolitana.

Palabras clave: Odontología forense, estimación de la edad, transparencia radicular.

\section{Abstract}

The aim of this study was to develop a specific dental age estimation formula for adults in Lima based on Lamendin's, Prince - Ubelaker's and Ubelaker - Parra's methods. The sample consisted in 50 healthy permanent one-root teeth of adult patients between 25 to 75 years old treated in health centers in Lima city. The applicability of the three dental methods (Lamendin, Prince - Ubelaker and Ubelaker-Parra) were validated to estimate the age of the sample. This was done using the Wilcoxon test that showed that the ages estimated by both, Prince - Ubelaker`s and Ubelaker - Parra`s methods did not have significant difference from the real age, compared with the results of Lamendin that actually showed significant differences. Age estimation was better with Prince - Ubelaker`s method $(r=0.935)$ compared with Ubelaker - Parra`s method $(r=0.918)$, so the first method was the most accurate to estimate the age of the sample. Applying the multiple regression test a new formula could be obtained, which is more accurate for age estimation in this adult population of Lima city. The conclusion was that Prince - Ublekar's and Ubelaker - Parra`s methods are valid for adult age estimation of Lima city, also a more specific dental method for age estimation in a sample of adult population of Lima City was develop.

Key words: Forensic dentistry, age estimation, root translucency.
Artículo Original

\section{Henry Jesús Vilcapoma Guerra}

Teniente FAP Odontólogo de la Sección de Odontología del Hospital Las Palmas.

Correspondencia:

Av. José Santos Chocano 338. Urb. Vil7la Los Ángeles. Los Olivos. Lima, Perú.

Telf: 989-636-8637

Correo electrónico:

henry_vilcapoma@hotmail.com
Fecha de recepción: 08-08-12

Fecha de aceptación: 16-10-12

\section{Introducción}

El desarrollo de la odontología forense durante los últimos años ha estado promovido por la situación actual de violencia que se presenta en muchos lugares del mundo, lo cual ha llevado a aumentar diariamente el número de personas fallecidas, al igual que el de cuerpos no identificados.

Cuerpos de miles de víctimas de conflicto armado, pertenecientes a la sociedad civil, soldados, guerrilleros y demás miembros, que mueren a causa de éste; o simplemente cuerpos de personas que fallecen de forma violenta o no, que las mismas condiciones de su muerte hacen difícil su identificación.

Sin embargo, la identificación humana no sólo es un objetivo de la odontología forense, sino que además sobre todo es una necesidad frente a un legítimo derecho individual y colectivo reconocido jurídicamente y, por ello, tratado como cualquier otro derecho fundamental, donde reza que: "Toda persona o comunidad tiene derecho a la memoria, a recordar y ser recordada sin distinciones ni discriminaciones de ningún tipo" ${ }^{1}$.

Para la identificación de un cadáver es necesario intentar determinar la mayor cantidad de parámetros posibles (la edad, el sexo, la estatura, la estimación del intervalo pos mortem); los cuales tienen un papel muy importante en las ciencias forenses, porque permitirá no sólo la identificación de los cuerpos, sino que será muy útil para la investigación en casos donde se sospeche que existe conexión con crímenes y/o accidentes ${ }^{2-6}$

Cuando los cadáveres se encuentran muy destruidos o deteriorados por el paso del tiempo o por cualquier otra circunstancia (efecto del fuego, humedad, el tiempo, etcétera), la determinación de estos parámetros puede ser complicada ${ }^{1,5}$. 
Actualmente, la mayoría de los métodos esqueléticos empleados para la estimación de la edad, establecen rangos de edad muy amplios y tienen un límite de edad superior que no sobrepasa (o lo hace en poco) los 45 ańos, por lo cual se tiene la necesidad de encontrar técnicas que sean más exactas o que por lo menos disminuyan dicho rango de edad.

Es por este motivo que el objetivo de la investigación es contribuir al proceso de identificación humana en el ámbito forense, para la estimación de la edad mediante los métodos dentales de Lamendin, Prince y Ubelaker y Ubelaker y Parra.

\section{Material y Método}

La investigación es transversal, prospectiva y comparativa. Se seleccionaron en forma no probabilística y por conveniencia 50 individuos adultos entre 25 y 75 ańos atendidos en Centros de Salud de Lima Metropolitana. La unidad de análisis fueron los dientes permanentes, uniradiculares, erupcionados completamente sanos, extraídos por motivos ortodónticos.

Luego de extraer los dientes en forma atraumática se midió la periodontosis (figura 1 y 2) a través del borde más coronal de la inserción periodontal hacia el límite amelocementario, medidos sobre la superficie bucal del diente. Para medir esta distancia el investigador debió estar presente en el momento de la cirugía. Las exodoncias se realizaron sin el uso de elevadores, buscando no alterar la altura de la periodontosis. En los casos donde se requirió el empleo de elevadores, el uso de éstos se aplicaron sobre las superficies proximales de los dientes ya que la altura de la periodontosis se midió por la superficie bucal.

Seguidamente los dientes fueron lavados e introducidos en hipoclorito sódico al $0.05 \%$ durante 5 minutos; después fueron secados, almacenados e identificados en bolsas plásticas. Pos-

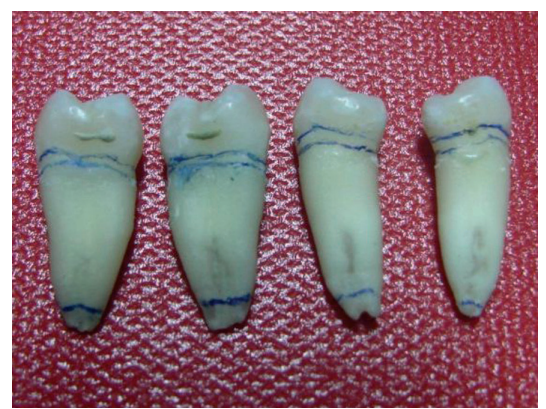

Fig 1. Delimitación de los parámetros a medir.

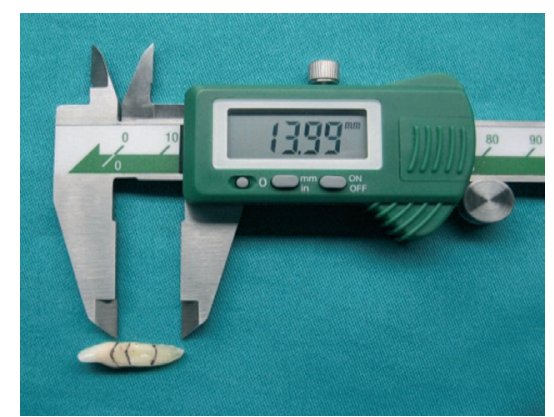

Fig 3. Altura de la parodontosis.

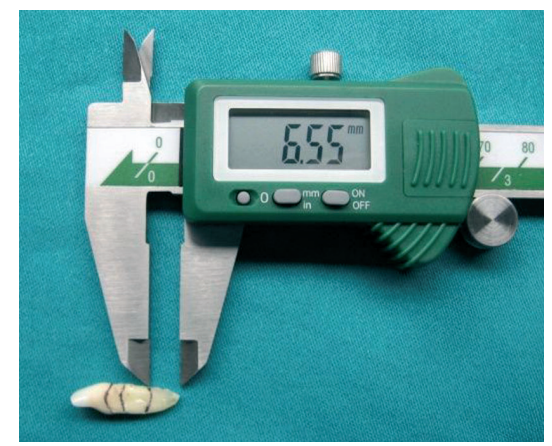

Fig 5. Medida de la transparencia radicular

teriormente se midieron la altura total de la raíz y la altura de la transparencia radicular (figuras 3,4,5 y 6).

Para la altura total de la raíz se midió la distancia entre el límite amelocementario hasta el ápice radicular. Estas medidas fueron tomadas sobre la superficie vestibular del diente, sin seccionarlo.

Para medir la altura de la transparencia, el diente fue colocado sobre un negatoscopio (compuesto por una lámpara de luz blanca de $40 \mathrm{~W}$ dentro de una caja, con una lámina de vinilo); se midió la altura de la transparencia desde el ápice radicular del diente, sobre la superficie vestibular y distal, tomando la extensión más alta de las dos, ya que, en algunas ocasiones la transparencia fue más alta en distal.

Todas las medidas se tomaron con un calibrador digital (marca: INSIZE y resolución: $0.01 \mathrm{~mm} / 0.0005)$ y fueron

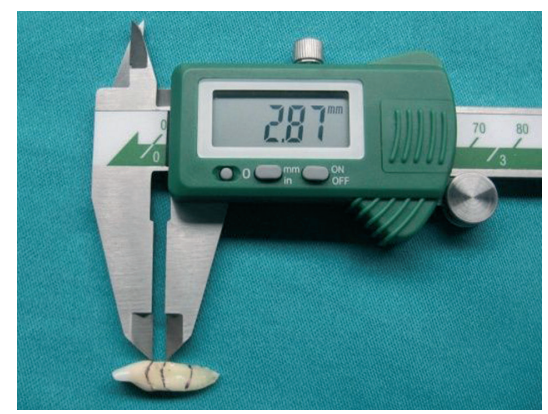

Fig 2. Medida de la altura de la periodontosis.

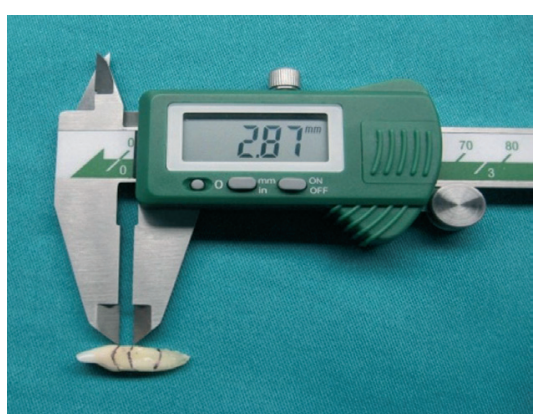

Fig 4. Medida de la longitud total de la raíz.

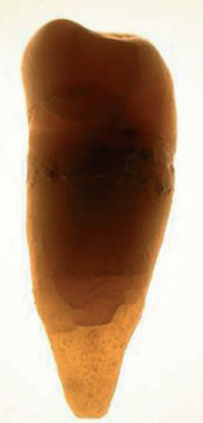

Fig 6. Transparencia radicular vista con negatoscopio

expresadas en décimas de milímetros (figura 6).

Para la estimación de las edades de las piezas dentarias de la muestra, se aplicaron las ecuaciones de Lamendin et al (1992), Prince y Ubelaker (2002) y Ubelaker y Parra (2008).

Para el procesamiento de los datos obtenidos, se elaboró una matriz de datos donde se registró: número de ficha, datos generales, ubicación del diente, tipo de diente, altura de periodontosis, altura de la transparencia radicular, longitud radicular, edad real y edad estimada por los métodos de Lamendin, Prince - Ubelaker y Ubelaker-Parra.

Para el análisis estadístico se usó el paquete estadístico SPSS v15.0. Primero, se halló el promedio de error de las edades estimadas por cada método con respecto a la edad real.

Luego, se empleó el Test de Wilcoxon para evaluar la aplicabilidad de cada uno de los tres métodos en la población de Lima Metropolitana para estimar la edad.

Para la elaboración de una fórmula dental específica para estimar la edad en una población de Lima Metropolitana, se realizó un análisis de regresión múltiple con los tres factores utilizados como determinantes de la edad según el método cuyos valores de las edades calculadas fueron más cercanos a los valores de las edades reales. 


\section{Resultados}

Las edades estimadas tanto por el método de Prince - Ubelaker como el de Ubelaker - Parra no difirieron significativamente de la edad real, en comparación con la de Lamendin que si tuvo una diferencia significativa. Sin embargo, la estimación fue mejor mediante el método de Prince - Ublekar ( $r=0.996)$, comparado con el de Ubelaker - Parra ( $\mathrm{r}=0.889)$ (Cuadro 1,2,3).

Por consiguiente el método según el cual las edades estimadas no llegaron a diferir significativamente de las edades reales o están más próximas a estas es el método de Prince - Ubelaker. Por lo tanto la nueva fórmula se obtendrá aplicando la prueba de regresión múltiple sobre la fórmula de Ubelaker - Parra.

\section{Prueba de Regresión Múltiple}

La regresión múltiple en este estudio sirvió para realizar un ajuste de la fórmula de Prince y Ubelaker para estimar la edad en la muestra de individuos adultos de Lima Metropolitana. Como variable dependiente (respuesta) la edad y variables Independientes (regresores): altura de la periodontosis, altura radicular y altura de la transparencia radicular. Se encontró que la variable más relacionada con la variable respuesta edad es la altura de la transparencia radicular 0.878, seguida de la altura de la periodontosis 0.819 y la altura radicular -0.478 . Se observó que no existe colinealidad entre variables independientes, ni correlación significativa.

En conclusión, a partir del análisis anterior se obtiene la siguiente fórmula:

$\mathrm{Edad}=55.06+2.78 \mathrm{AP}-2.02 \mathrm{AR}+$ 2.85 AT

Donde

$\mathrm{AP}=$ Altura de la periodontosis

\section{$\mathrm{AR}=$ Altura radicular}

AT $=$ Altura de la transparencia radicular

En Tabla 1 se observa el promedio de error en la estimación de la edad usando la nueva fórmula. El promedio de error se refiere a la diferencia entre la edad estimada y la edad real. Para el caso de la muestra de individuos adultos de Lima Metropolitana estudiada en esta investigación, con la nueva fórmula se obtiene un mínimo promedio de error de 3.7 años.

Tabla 1. Análisis del promedio de error en la estimación de la edad de individuos adultos de Lima Metropolitana usando la nueva fórmula.

\begin{tabular}{ccc}
\hline Método & Promedio de error & Desviación estándar \\
\hline Lamendin & 5.6 & 5.61423419 \\
Prince y Ubelaker & 5.49 & 5.018377041 \\
Ubelaker y Parra & 7.01 & 4.24176 \\
Vilcapoma & 3.7 & 3.53360 \\
\hline
\end{tabular}

\section{Discusión}

El método de Prince y Ubelaker toma en cuenta los mismos parámetros que Lamendin, pero cuestiona el uso del método de Lamendin para poblaciones diferentes a la muestra francesa que el empleó ${ }^{8}$. Migyesi encontró un promedio de error 8.2años con el uso del método de Prince y Ubelaker para una muestra inglesa, valor mucho menor que el hallado con el método de Lamendin 9. Por su parte Gonzáles también reportó un promedio de error menor con el empleo del método de Prince y Ubelaker (0.33 años) comparado al método de Lamendin en una población española ${ }^{11}$. En una población peruana, Ubelaker y Parra también reportaron un promedio de error de 7.62 años, menor al compararlo con el hallado con el método de Lamendin ${ }^{12}$. Éste último resultado guarda mucha similitud a los resultados hallados en esta investigación donde al emplear el método de Prince y Ubelaker en una población peruana (Lima Metropolitana) se obtuvo un promedio de error de 7.49 años.

En el Perú la tendencia de hallar modelos o fórmulas dentales específicas para estimar la edad llega en el año 2008 con el trabajo de Ubelaker y Parra. En su estudio reportan un promedio de error de su método de 6.28 años $^{12}$. En este trabajo se obtuvo un valor cercano de 7.01 años. Esta diferencia podría ser debido al empleo de una muestra diferente, ya que en este estudio la muestra fue de individuos que radican en Lima Metropolitana, mientras que en el estudio de Ubelaker y Parra la muestra la conformaron restos cadavéricos de individuos de diferentes departamentos del Perú.

La validación o aplicabilidad de los diferentes métodos para estimar la edad en una población determinada se refiere a verificar si existe o no diferencia significativa entre los valores de las edades estimadas y los valores de las edades reales. Gonzales reportó una diferencia significativa de esos valores al usar el método de Lamendin para una muestra española, mientras que usando el método de Prince y Ubelaker no había diferencia significativa entre esos valores. Por otro lado, al aplicar tanto el método de Lamendin como el de Prince - Ubelaker no había diferencia signigicativa con la edad real para una muestra colombiana ${ }^{11}$. Dentro de los resultados de esta investigación, se mostró que existe diferencia significativa entre los valores de las edades estimadas con el método de Lamendin y los valores de las edades reales. Sin embargo, usando los métodos de Prince - Ubelaker y Ubelaker Parra no existió diferencia significativa con los valores de las edades reales.

$\mathrm{Al}$ igual que Mastrille ${ }^{10}$ y Baccino $^{7}$ consideramos que el empleo en conjunto de varios métodos para estimar la edad ofrece resultados más exactos que el empleo únicamente de un método dental.

En los estudios de Prince y Ubelaker ${ }^{8}$, Gonzáles $^{11}$ y Ubelaker y Parra $^{12}$ se desarrollaron nuevas fórmulas específicas para un determinado grupo poblacional, los cuales lograron estimar la edad con mayor exactitud. En esta investigación, de la misma manera, se desarrolló una fórmula modificada a partir de la fórmula de Prince-Ubelaker la cual mostró resultados más cercanos a la edad real para una muestra de individuos de Lima Metropolitana.

En lo referente al ajuste del método de Prince y Ubelaker empleando la prueba de regresión múltiple para estimar la edad de la muestra de individuos adultos de Lima Metropolitana, se obtuvo a partir del análisis de correlación que la mayor correlación de la edad con la transparencia radicular $(r=0.878)$. Este resultado de correlación concuerda con el hallado por Gonzáles para un muestra Colombiana $(\mathrm{r}=0.65-0.87)^{11}$.

Tras la validación del modelo obtenido con el análisis de Varianza (ANOVA) se obtuvo que el modelo de regresión múltiple fue significativo para la muestra 
del estudio, por lo cual no fue necesario realizar un ajuste posterior y el modelo obtenido inicialmente fue suficiente.

\section{Conclusiones}

Se concluye que el método de Lamendin no fue válido para estimar la edad en la muestra de individuos adultos de Lima Metropolitana. Los métodos de Ubelaker - Parra y de Prince - Ubelaker fueron válidos para estimar la edad en dicha muestra, siendo este último más preciso. Fue factible desarrollar una fórmula dental específica para la estimación de la edad basados en los método de Lamendin, Prince - Ubelaker y Ubelaker - Parra, en la muestra de individuos adultos de los Centros de Salud de Lima Metropolitana, en el año 2009 con lo cual se demostró la hipótesis de trabajo.

\section{Referencias bibliográficas}

1. Gobierno de la república de Colombia. Fundación Manuel Cepeda Vargas [Internet]. 2003: [acceso 1 de diciembre del 2009]: [aprox. 5p]. Disponible en: http://www. desaparecidos-org/colombia/galeria
2. Ubelaker D. Human skeletal remains. $2^{\text {a }}$ ed. Washington: Smithsonian Institution; 1996.

3. Delgado C. Aplicación de la antropología forense dentro del derecho internacional humanitario. El caso de conflicto armado en Colombia. Ciudad invertial de la antropología y arquectomía. Bogotá; 2002.

4. Krogman W, Iscan M. The human skeleton in forensic medicine. Springfield; Charles Tomas; 1986.

5. Sanabria C. Antropología forense y la investigación médico - legal de las muertes. $1^{a}$ ed. Bogotá: Facultad de Investigación Criminal. Policia Nacional; 2004.

6. Lamendin H, Baccino E, Humbert J, Tavernier J, Nossintchouk R, y Serilli A. A simple technique for age estimation in adult corpses: The two criteria dental method. Journal of Forensic Sciences. 1992; 37 (5): $1373-9$.

7. Baccino E, Ubelaker D, Hayek. L y Zerilli A. Evaluation of seven methods of estimating age at death from mature human skeletal remains. Journal of Forensic Sciences. 1999; 44 (E): 931 - 6.
8. Prince D y Ubelaker D. Application of Lamendin-s adult dental aging technique to a diverse skeletal simple. Journal of Forensic Sciences. 2002; 47 (1): 107 - 16.

9. Migyesi M, Ubelaker D y Sauer N. Test of the Lamendin aging method on two historic skeletal samples. American Journal of Physical Antropology. 2006; 131: $363-7$.

10. Mastrille L, Ubelaker D, Cattaneo C, Seguret F, Tremblay M, Barcino E. Comparison of four skeletal methods for the estimates of age at death on White and Black Adults. Journal of Forensic Sciences. 2007; 52(2): $115-9$.

11. Gonzáles G. Determinación de la edad en adultos mediante un método dental. Aplicación y análisis. [Tesis Doctoral] Granada; Facultad de Medicina, Universidad de Granada. 2007.

12. Ubelaker D, Parra R. Application of three dental methods of adult age estimation from intact single rooted teeth to a Peruvian Sample. Journal of Forensic Sciences. 2008; 53(3): $608-11$. 